\title{
Polymorphism in Zintl-Phases $\mathrm{ACd}_{4} \mathrm{Pn}_{3}$ : The modulated structures of $\mathrm{NaCd}_{4} P n_{3}$ with $P n=\mathrm{P}$, As
}

Carolin Grotz, Maximilian Baumgartner, Katharina M. Freitag, Franziska Baumer, Tom Nilges*

Department of Chemistry, Synthesis and Characterization of Innovative Materials, Technical University Munich, Lichtenbergstraße 4, 85747 Garching, Germany 


\section{Powder pattern and DSC measurement of $\mathrm{NaCd}_{4} \mathrm{P}_{3}$}

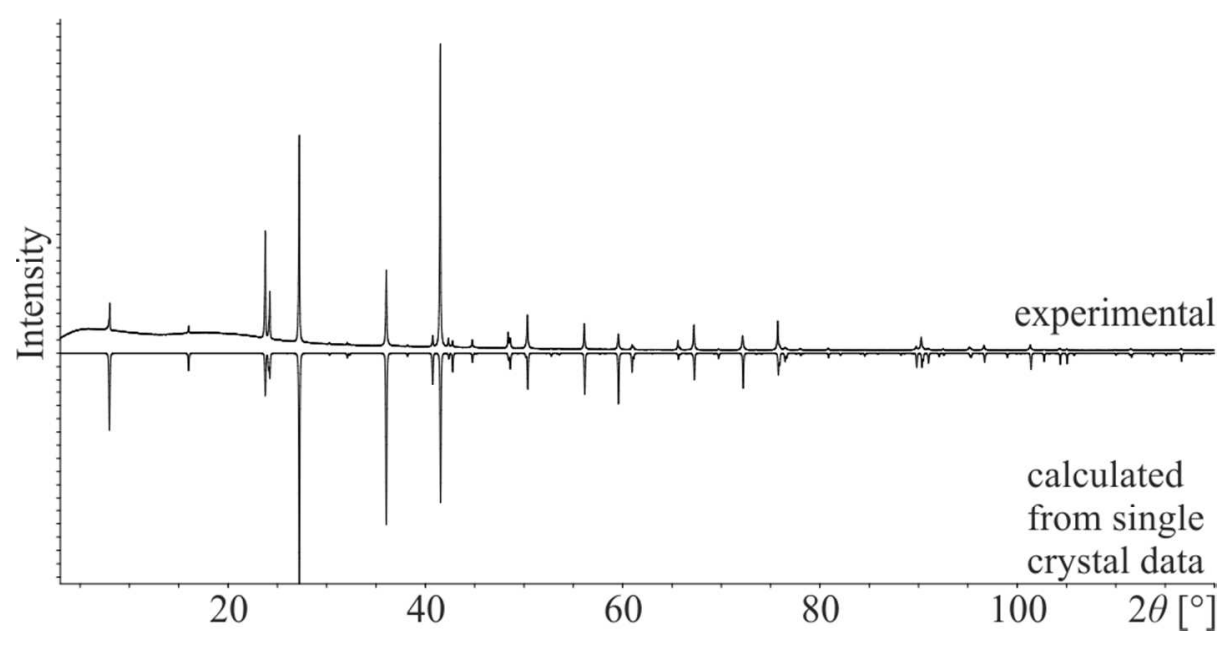

Figure S1. Experimental (above) and theoretical (calculated from single crystal data) powder patterns of $\beta$ - $\mathrm{NaCd}_{4} \mathrm{P}_{3}$, measured at room temperature. Texture effects are visible due to the morphology of the crystals.

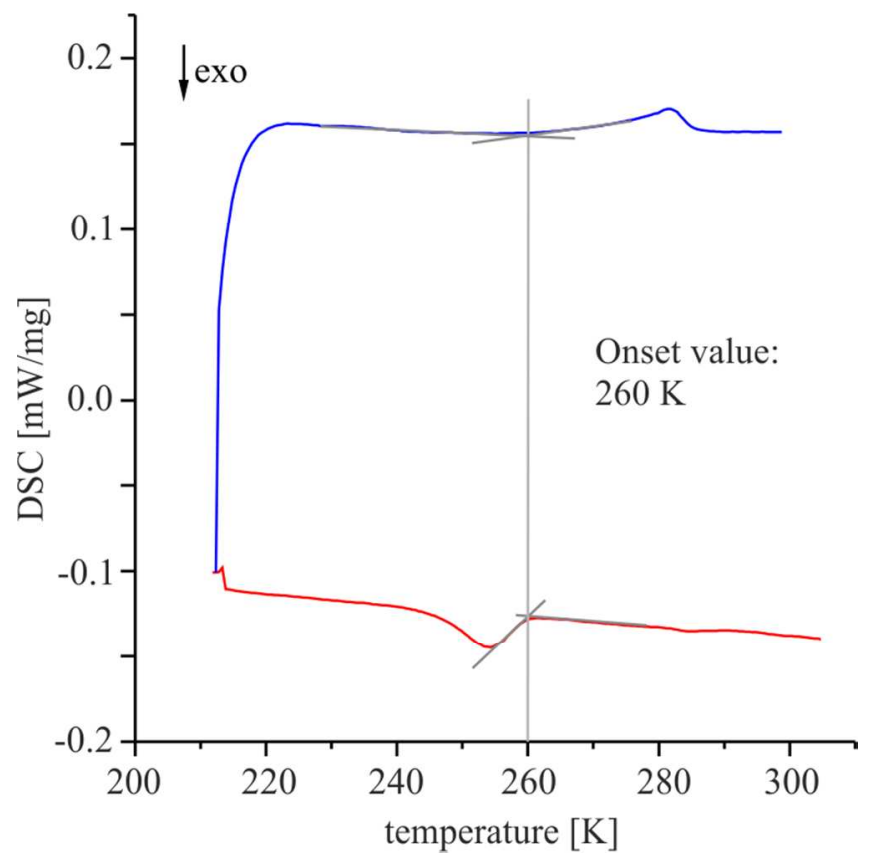

Figure S2. Third cycle of a DSC measurement of $\mathrm{NaCd}_{4} \mathrm{P}_{3}$ (red: cooling section; blue: heating section). The grey lines are illustrating the onset value of a reversible phase transition at $260 \mathrm{~K}$. No additional signals were observed in the temperature range between 113 and $333 \mathrm{~K}$. 


\section{Rietveld analysis of $\beta-\mathrm{NaCd}_{4} \mathrm{P}_{3}$ and $\beta-\mathrm{NaCd}_{4} \mathrm{As}_{3}$ at room temperature}

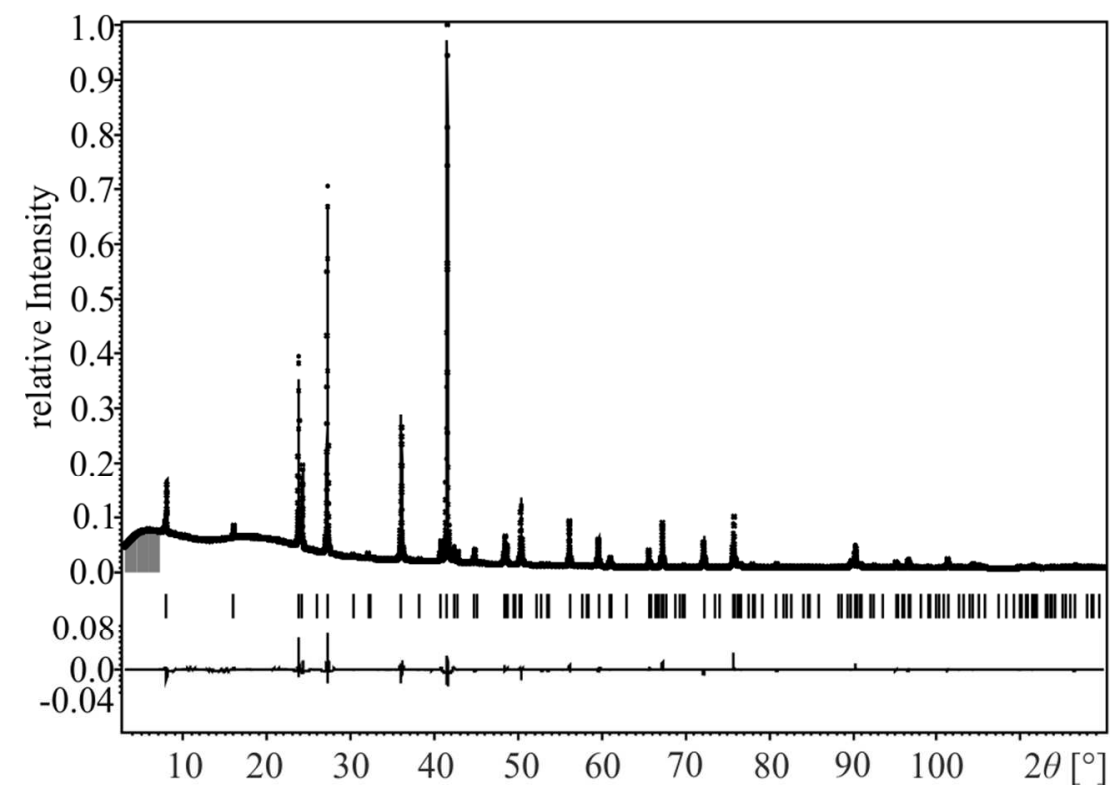

Figure S3. Rietveld refinement of X-ray powder data of $\beta-\mathrm{NaCd}_{4} \mathrm{P}_{3}$, measured at room temperature $(R \overline{3} m$, above) and difference plot $I(\mathrm{obs})-I($ calc) (below). The grey area was rejected from calculation.

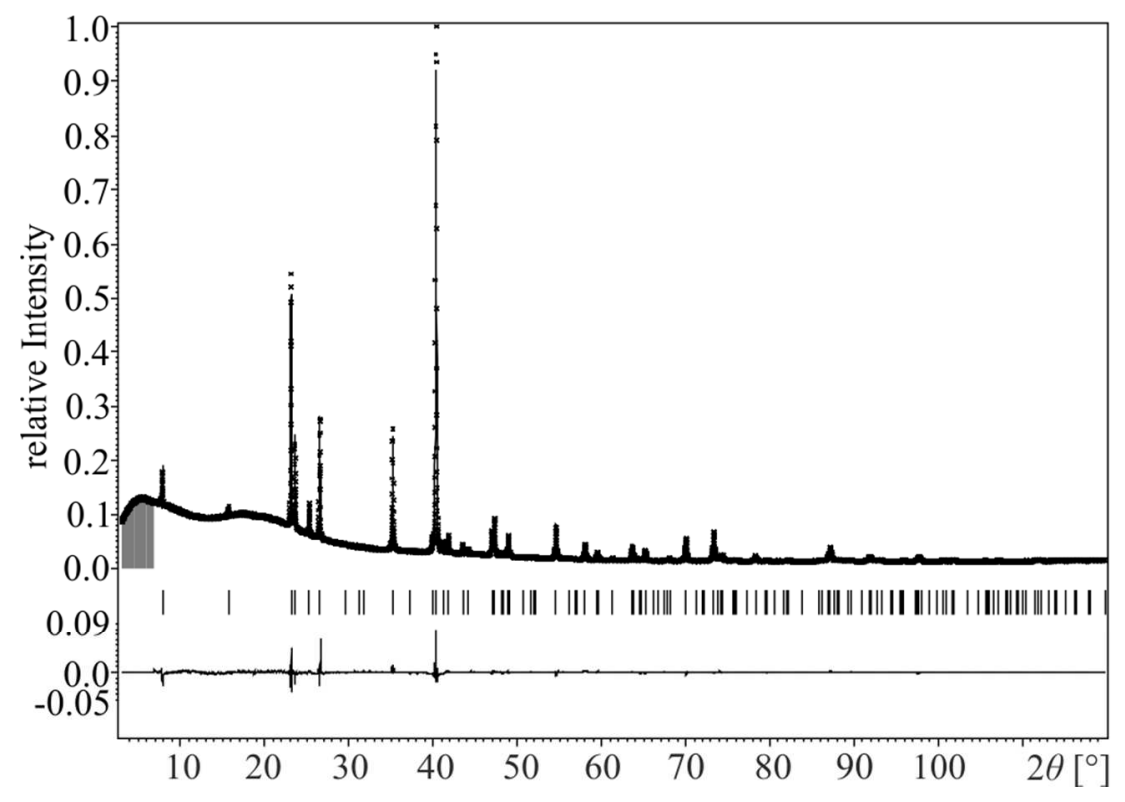

Figure S4. Rietveld refinement of X-ray powder data of $\beta-\mathrm{NaCd}_{4} \mathrm{As}_{3}$, measured at room temperature $(R \overline{3} m$, above) and difference plot $I(\mathrm{obs})-I($ calc) (below). The grey area was rejected from calculation. 
Table S1. Crystallographic data of $\beta-\mathrm{NaCd}_{4} \mathrm{Pn}_{3}$, derived from Rietveld refinements of X-ray powder data at room temperature (space group: $R \overline{3} m$ ).

\begin{tabular}{|c|c|c|}
\hline & $\beta-\mathrm{NaCd}_{4} \mathrm{P}_{3}$ & $\beta-\mathrm{NaCd}_{4} \mathrm{As}_{3}$ \\
\hline Temperature $[\mathrm{K}]$ & 293 & 293 \\
\hline Refined formula & $\mathrm{NaCd}_{4} \mathrm{P}_{3}$ & $\mathrm{NaCd}_{4} \mathrm{As}_{3}$ \\
\hline Molecular weight $[\mathrm{g} / \mathrm{mol}]$ & 565.6 & 697.4 \\
\hline Crystal system & rhombohedral & Rhombohedral \\
\hline Space group & $R \overline{3} m($ no. 166$)$ & $R \overline{3} m($ no. 166$)$ \\
\hline \multicolumn{3}{|l|}{ Unit cell dimension } \\
\hline$a[\AA]$ & $4.34713(3)$ & $4.46825(2)$ \\
\hline$c[\AA]$ & $33.1949(4)$ & $33.8406(5)$ \\
\hline cell volume $\left[\AA^{3}\right]$ & $543.259(8)$ & $585.12(1)$ \\
\hline$Z$ & 3 & 3 \\
\hline Calcd density $\left[\mathrm{g} / \mathrm{cm}^{3}\right]$ & 5.19 & 5.94 \\
\hline$\theta$ range $[\mathrm{deg}]$, increment $[\mathrm{deg}]$ & $3.000-119.955,0.015$ & $3.000-119.880,0.015$ \\
\hline Abs coeff $\left[\mathrm{mm}^{-1}\right]$ & 101.5 & 102.6 \\
\hline Data collection & Stoe STADI P (mythen 1K) & Stoe STADI P (mythen 1K) \\
\hline Radiation & $\mathrm{Cu}-\mathrm{K}_{\alpha 1}(1.5405 \AA), \mathrm{Ge}$ & $\mathrm{Cu}-\mathrm{K}_{\alpha 1}(1.5405 \AA), \mathrm{Ge}$ \\
\hline No. data points & 7798 & 7793 \\
\hline No. of parameter & 34 & 34 \\
\hline Profil function & Pseudo-Voigt & Pseudo-Voigt \\
\hline Background & 17 Legendre polynoms & 17 Legendre polynoms \\
\hline Preferred orientation & March \& Dollase & March \& Dollase \\
\hline GOF on $\mathrm{F}^{2}$ & 2.17 & 1.73 \\
\hline $\mathrm{R}_{\mathrm{p}} / \mathrm{wR}_{\mathrm{p}}$ & $0.0287 / 0.0439$ & $0.0242 / 0.0369$ \\
\hline R1 / wR2 (all data) & $0.0690 / 0.0586$ & $0.0714 / 0.0589$ \\
\hline Largest diff peak/hole $\left[\mathrm{e} / \AA^{3}\right]$ & $2.64 /-2.24$ & $2.89 /-2.26$ \\
\hline
\end{tabular}


Table S2. Crystal structure parameter and atomic displacements $\left(\AA^{2}\right)$, derived from Rietveld refinements of X-ray powder data of $\beta-\mathrm{NaCd}_{4} \mathrm{P}_{3}$, measured at room temperature. Wyckoff position, atomic coordinates, isotropic and anisotropic displacement parameters $\left(\AA^{2}\right)$ are given (space group: $R \overline{3} \mathrm{~m}$ ). The anisotropic displacement factor exponent takes the form: $-2 \pi^{2}\left[\left(\mathrm{ha}^{*}\right) 2 U_{11}+\ldots+2 \mathrm{hka} \cdot \mathrm{b} \cdot U_{12}\right] . U_{e q}$ is defined as one third of the trace of the orthogonalized $U_{i j}$ tensor. * Displacement was refined isotropically only.

\begin{tabular}{|c|c|c|c|c|c|c|}
\hline$\beta-\mathrm{NaCd}_{4} \mathrm{P}_{3}$ & \multicolumn{2}{|c|}{ Wyckoff position } & $x$ & $y$ & $z$ & $U_{i s s}{ }^{*} / U_{e q}$ \\
\hline Cd1 & \multicolumn{2}{|c|}{$6 c$} & 0 & 0 & $0.09808(7)$ & $0.0390(9)$ \\
\hline P1 & \multicolumn{2}{|c|}{$6 c$} & 0 & 0 & $0.2214(2)$ & $0.013(2)$ \\
\hline $\mathrm{Cd} 2$ & \multicolumn{2}{|c|}{$6 c$} & 0 & 0 & $0.29895(7)$ & $0.0338(7)$ \\
\hline $\mathrm{Na} 1$ & \multicolumn{2}{|c|}{$3 b$} & 0 & 0 & $1 / 2$ & $0.041(3) *$ \\
\hline \multirow[t]{2}{*}{$\mathrm{P} 2$} & \multicolumn{2}{|c|}{$3 a$} & 0 & 0 & 0 & $0.012(2)^{*}$ \\
\hline & $U_{11}$ & $U_{22}$ & $U_{33}$ & $U_{12}$ & $U_{13}$ & $U_{23}$ \\
\hline Cd1 & $0.0154(4)$ & $0.0154(4)$ & $0.086(2)$ & $0.0077(2)$ & 0 & 0 \\
\hline P1 & $0.005(1)$ & $0.005(1)$ & $0.030(6)$ & $0.0024(7)$ & 0 & 0 \\
\hline $\mathrm{Cd} 2$ & $0.0498(6)$ & $0.0498(6)$ & $0.002(2)$ & $0.0249(3)$ & 0 & 0 \\
\hline
\end{tabular}

Table S3. Crystal structure parameter and atomic displacements $\left(\AA^{2}\right)$, derived from Rietveld refinements of X-ray powder data of $\beta-\mathrm{NaCd}_{4} \mathrm{As}_{3}$, measured at room temperature. Wyckoff position, atomic coordinates, isotropic and anisotropic displacement parameters $\left(\AA^{2}\right)$ are given (space group: $R \overline{3} m$ ). The anisotropic displacement factor exponent takes the form: $2 \pi^{2}\left[\left(\mathrm{ha}^{*}\right) 2 U_{11}+\ldots+2 \mathrm{hka} \cdot \mathrm{b} \cdot U_{12}\right] . U_{e q}$ is defined as one third of the trace of the orthogonalized $U_{i j}$ tensor. * Displacement was refined isotropically only.

\begin{tabular}{|c|c|c|c|c|c|c|}
\hline & \multicolumn{2}{|c|}{ Wyckoff position } & $x$ & $y$ & $z$ & $U_{i s o}{ }^{*} / U_{e q}$ \\
\hline Cd1 & \multicolumn{2}{|c|}{$6 c$} & 0 & 0 & $0.09530(8)$ & $0.0265(9)$ \\
\hline As1 & \multicolumn{2}{|c|}{$6 c$} & 0 & 0 & $0.2195(1)$ & $0.012(1)$ \\
\hline $\mathrm{Cd} 2$ & \multicolumn{2}{|c|}{$6 c$} & 0 & 0 & $0.29955(9)$ & $0.0461(7)^{*}$ \\
\hline $\mathrm{Na} 1$ & \multicolumn{2}{|c|}{$3 b$} & 0 & 0 & 0.5 & $0.018(3)^{*}$ \\
\hline \multirow[t]{2}{*}{ As2 } & \multicolumn{2}{|c|}{$3 a$} & 0 & 0 & 0 & $0.026(2)$ \\
\hline & $U_{11}$ & $U_{22}$ & $U_{33}$ & $U_{12}$ & $U_{13}$ & $U_{23}$ \\
\hline $\mathrm{Cd} 1$ & $0.0234(5)$ & $0.0234(5)$ & $0.033(3)$ & $0.0117(3)$ & 0 & 0 \\
\hline As 1 & $0.0151(8)$ & $0.0151(8)$ & $0.005(3)$ & $0.0076(4)$ & 0 & 0 \\
\hline As2 & $0.024(1)$ & $0.024(1)$ & $0.031(6)$ & $0.0118(7)$ & 0 & 0 \\
\hline
\end{tabular}


3. Room temperature single crystal $X$-ray structure data of $\beta-\mathrm{NaCd}_{4} \mathrm{P}_{3}$

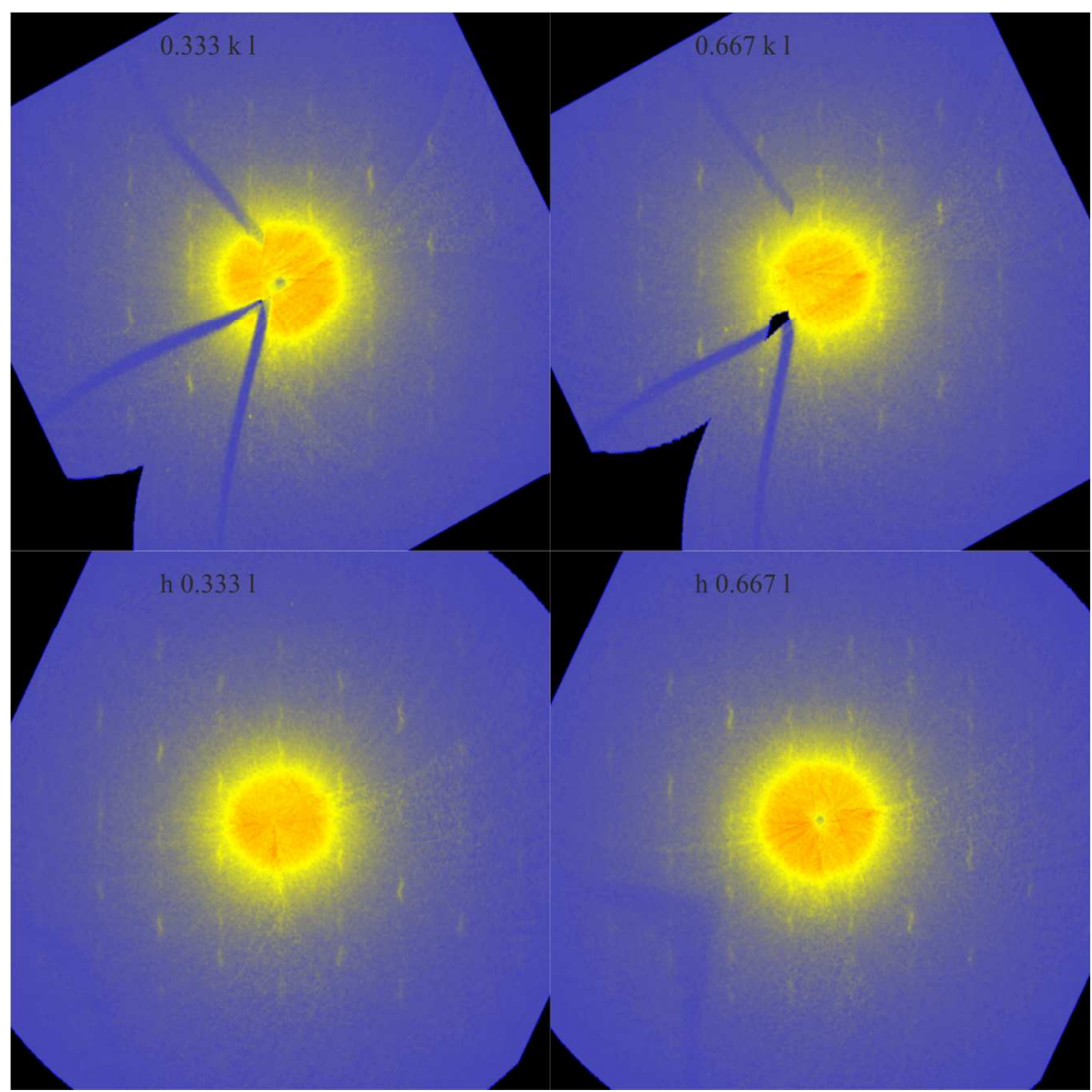

Figure S5. Reciprocal space representation of $\beta-\mathrm{NaCd}_{4} \mathrm{P}_{3}$ derived from room temperature data. The rhombohedral cell with cell parameters $a=4.3445(5) \AA, c=33.197(5) \AA$ (space group: $R \overline{3} \mathrm{~m})$ was used to create these pictures. Wave-like diffuse scattering intensities are present at $(0.333 k l),(0.667 k l),(h 0.333 l)$, and $(h 0.667 l)$ sections. All diffuse intensity is neglected in the room temperature refinements. 
Table S4. Crystal structure parameter and atomic displacements $\left(\AA^{2}\right)$, derived from X-ray single crystal data of $\beta-\mathrm{NaCd}_{4} \mathrm{P}_{3}$, measured at room temperature. Wyckoff position, atomic coordinates and anisotropic displacement parameters $\left(\AA^{2}\right)$ for $\mathrm{NaCd}_{4} \mathrm{P}_{3}$ are given (space group: $R \overline{3} \mathrm{~m}$ ). The anisotropic displacement factor exponent takes the form: $-2 \pi^{2}\left[\left(\mathrm{ha}^{*}\right) 2 U_{11}+\ldots+2 \mathrm{hka} \cdot \mathrm{b} \cdot U_{12}\right] . U_{e q}$ is defined as one third of the trace of the orthogonalized $U_{i j}$ tensor.

\begin{tabular}{|c|c|c|c|c|c|c|}
\hline$\beta-\mathrm{NaCd}_{4} \mathrm{P}_{3}$ & \multicolumn{2}{|c|}{ Wyckoff position } & $x$ & $y$ & $z$ & $U_{e q}$ \\
\hline $\mathrm{Cd} 1$ & \multicolumn{2}{|c|}{$6 c$} & 0 & 0 & $0.09768(6)$ & $0.0324(6)$ \\
\hline P1 & \multicolumn{2}{|c|}{$6 c$} & 0 & 0 & $0.2221(1)$ & $0.010(1)$ \\
\hline $\mathrm{Cd} 2$ & \multicolumn{2}{|c|}{$6 c$} & 0 & 0 & $0.29950(4)$ & $0.0313(6)$ \\
\hline $\mathrm{Na} 1$ & \multicolumn{2}{|c|}{$3 b$} & 0 & 0 & $1 / 2$ & $0.029(3)$ \\
\hline P2 & \multicolumn{2}{|c|}{$3 a$} & 0 & 0 & 0 & $0.015(2)$ \\
\hline & $\overline{U_{11}}$ & $U_{22}$ & $U_{33}$ & $U_{12}$ & $U_{13}$ & $U_{23}$ \\
\hline Cd1 & $0.0120(6)$ & $0.0120(6)$ & $0.073(1)$ & $0.0060(3)$ & 0 & 0 \\
\hline P1 & $0.010(1)$ & $0.010(1)$ & $0.011(2)$ & $0.0047(7)$ & 0 & 0 \\
\hline $\mathrm{Cd} 2$ & $0.0405(7)$ & $0.0405(7)$ & $0.0130(7)$ & $0.0202(4)$ & 0 & 0 \\
\hline $\mathrm{Na} 1$ & $0.027(4)$ & $0.027(4)$ & $0.034(7)$ & $0.013(2)$ & 0 & 0 \\
\hline P2 & $0.011(2)$ & $0.011(2)$ & $0.023(3)$ & $0.0056(9)$ & 0 & 0 \\
\hline
\end{tabular}

Table S5. Selected interatomic distances $(\AA)$ derived from single crystal X-ray data of $\beta$ $\mathrm{NaCd}_{4} \mathrm{P}_{3}$, measured at room temperature (space group: $R \overline{3} \mathrm{~m}$ ). All distances of the first coordination sphere including standard deviations are given.

\begin{tabular}{|c|c|c|c|c|c|c|c|}
\hline Atom 1 & & Atom 2 & Distance $[\AA]$ & Atom 1 & & Atom 2 & Distance $[\AA]$ \\
\hline \multirow[t]{3}{*}{$\mathrm{Cd} 1$} & 3 & P1 & $2.5486(8)$ & $\mathrm{Cd} 2$ & & $\mathrm{P} 1$ & $2.571(4)$ \\
\hline & & P2 & $3.243(2)$ & & 3 & P2 & $2.7483(6)$ \\
\hline & 3 & $\mathrm{Na} 1$ & $3.397(1)$ & $\mathrm{Na} 1$ & 6 & P1 & $3.110(2)$ \\
\hline \multirow[t]{3}{*}{ P1 } & 3 & $\mathrm{Cd} 1$ & $2.5486(8)$ & & 6 & $\mathrm{Cd} 1$ & $3.397(1)$ \\
\hline & & $\mathrm{Cd} 2$ & $2.571(4)$ & P2 & 6 & $\mathrm{Cd} 2$ & $2.7483(6)$ \\
\hline & 3 & $\mathrm{Na} 1$ & $3.110(2)$ & & 2 & $\mathrm{Cd} 1$ & $3.243(2)$ \\
\hline
\end{tabular}




\section{Low temperature single crystal $X$-ray structure data of $\alpha-\mathrm{NaCd}_{4} \mathrm{P}_{3}$}

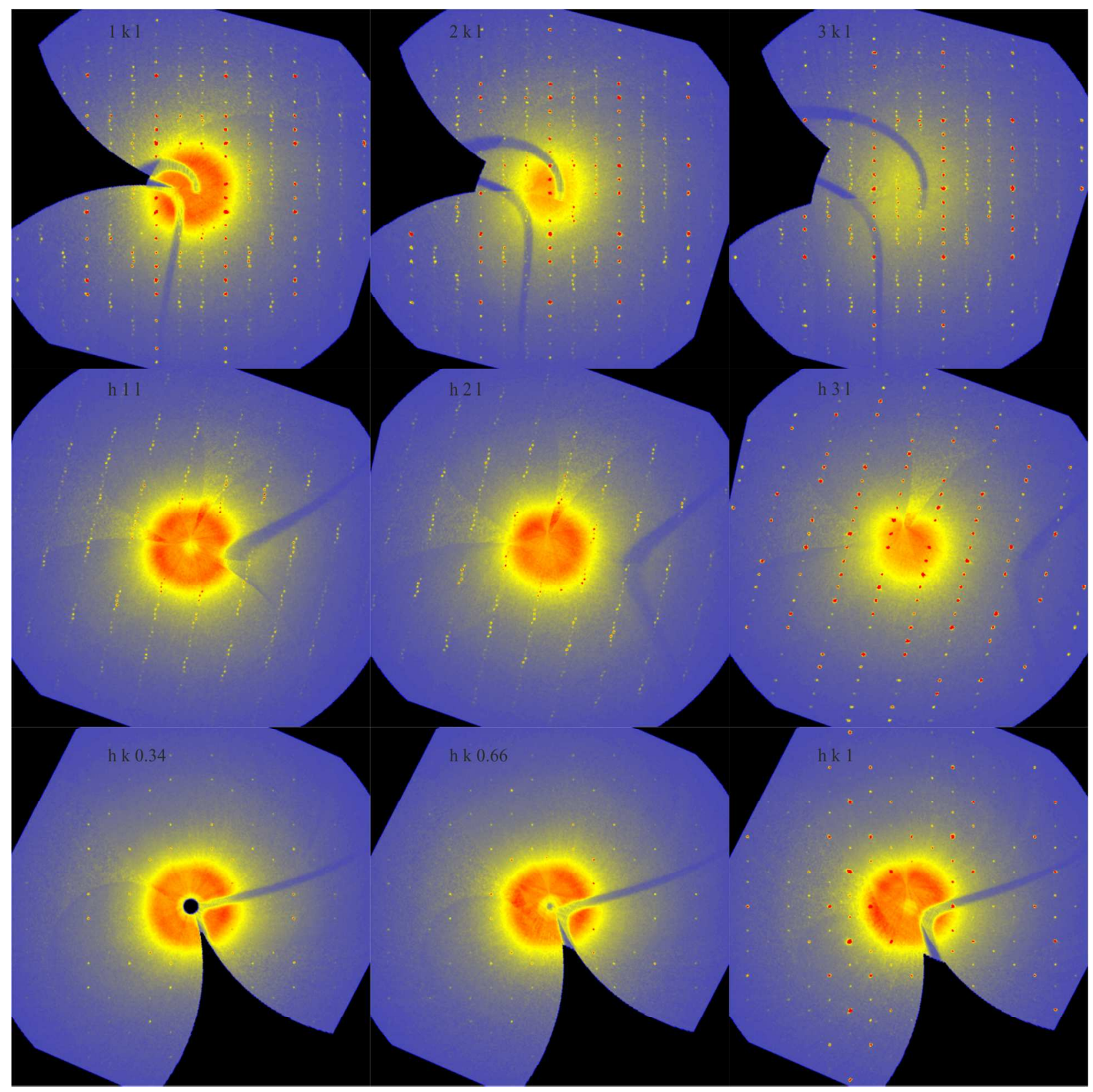

Figure S6. Reciprocal space representation of $\alpha-\mathrm{NaCd}_{4} \mathrm{P}_{3}$ derived from low temperature data $(130 \mathrm{~K})$. A monoclinic cell with cell parameters $a=7.5098(8) \AA, b=13.007(1) \AA, c=11.289(1)$ $\AA, \beta=102.827(8)^{\circ}$ was used to create these pictures. Areas of diffuse scattering which were present at room temperature (see Figure S5) show discrete Bragg reflections now. 
Table S6. Crystal structure parameters and atomic displacements $\left(\AA^{2}\right)$, derived from X-ray single crystal data of $\alpha-\mathrm{NaCd}_{4} \mathrm{P}_{3}$, measured at low temperature (130 K). Wyckoff position, atomic coordinates, isotropic and anisotropic displacement parameters $\left(\AA^{2}\right)$ for $\alpha-\mathrm{NaCd}_{4} \mathrm{P}_{3}$ are given (space group $\mathrm{Cm}(\alpha 0 \gamma) s)$. The anisotropic displacement factor exponent takes the form: $-2 \pi^{2}\left[\left(\mathrm{ha}^{*}\right) 2 U_{11}+\ldots+2 \mathrm{hka} \cdot \mathrm{b} \cdot U_{12}\right] . U_{e q}$ is defined as one third of the trace of the orthogonalized $U_{i j}$ tensor. * Displacement was refined isotropically only. ** Displacement parameter fixed.

\begin{tabular}{|c|c|c|c|c|c|c|}
\hline$\alpha-\mathrm{NaCd}_{4} \mathrm{P}_{3}$ & \multicolumn{2}{|c|}{ Wyckoff position } & $x$ & $y$ & $z$ & $U_{i s o} / U_{e q}$ \\
\hline $\mathrm{Cd} 1$ & \multicolumn{2}{|c|}{$4 b$} & $0.1767(3)$ & $0.1661(2)$ & $0.0315(2)$ & $0.0216(6)$ \\
\hline $\mathrm{Cd} 2$ & \multicolumn{2}{|c|}{$4 b$} & $0.3729(3)$ & $0.1670(2)$ & $0.6203(2)$ & $0.0220(6)$ \\
\hline $\mathrm{Cd} 3$ & \multicolumn{2}{|c|}{$2 a$} & $0.6842(5)$ & 0 & $0.0525(3)$ & $0.0153(7)$ \\
\hline $\mathrm{Cd} 4$ & \multicolumn{2}{|c|}{$2 a$} & $0.8809(6)$ & 0 & $0.6409(4)$ & $0.0229(9)$ \\
\hline $\mathrm{Cd} 5$ & \multicolumn{2}{|c|}{$4 b$} & $0.0689(3)$ & $0.3257(2)$ & $0.2300(2)$ & $0.0214(5)$ \\
\hline Cd6 & \multicolumn{2}{|c|}{$4 b$} & $0.4707(3)$ & $0.3403(3)$ & $0.4339(2)$ & $0.0195(5)$ \\
\hline $\mathrm{Cd} 7$ & \multicolumn{2}{|c|}{$2 a$} & $0.0921(5)$ & 0 & $0.2298(4)$ & $0.0221(7)$ \\
\hline $\mathrm{Cd} 8$ & \multicolumn{2}{|c|}{$2 a$} & $0.4926(5)$ & 0 & $0.4325(4)$ & $0.0207(7)$ \\
\hline P1 & \multicolumn{2}{|c|}{$4 b$} & $0.2767(9)$ & $0.1665(7)$ & $0.3312(7)$ & $0.012(1)$ \\
\hline P2 & \multicolumn{2}{|c|}{$2 a$} & $0.7744(9)$ & 0 & $0.3197(7)$ & $0.009(2)$ \\
\hline P3 & \multicolumn{2}{|c|}{$4 b$} & $0.0019(8)$ & $0.3330(5)$ & $0.0005(5)$ & $0.0015(9)^{*}$ \\
\hline P4 & \multicolumn{2}{|c|}{$4 b$} & $0.060(1)$ & $0.1683(5)$ & $0.6720(7)$ & $0.015(1)$ \\
\hline P5 & \multicolumn{2}{|c|}{$2 a$} & $0.001(2)$ & 0 & $0.000(1)$ & $0.003(2)^{*}$ \\
\hline P6 & \multicolumn{2}{|c|}{$2 a$} & $0.556(2)$ & 0 & $0.669(1)$ & $0.016(2)$ \\
\hline $\mathrm{Na} 1$ & \multicolumn{2}{|c|}{$4 b$} & $0.269(2)$ & $0.334(2)$ & $0.8353(9)$ & $0.015(1)$ \\
\hline \multirow[t]{2}{*}{$\mathrm{Na} 2$} & \multicolumn{2}{|c|}{$2 a$} & $0.288(2)$ & 0 & $0.835(1)$ & $0.02 * *$ \\
\hline & $U_{11}$ & $U_{22}$ & $U_{33}$ & $U_{12}$ & $U_{13}$ & $U_{23}$ \\
\hline $\mathrm{Cd} 1$ & $0.0107(8)$ & $0.0095(8)$ & $0.047(1)$ & $0.0004(7)$ & $0.0120(7)$ & $0.0008(8)$ \\
\hline $\mathrm{Cd} 2$ & $0.0124(8)$ & $0.0126(8)$ & $0.042(1)$ & $0.0002(7)$ & $0.0085(7)$ & $0.0010(9)$ \\
\hline $\mathrm{Cd} 3$ & $0.006(1)$ & $0.002(1)$ & $0.039(1)$ & 0 & $0.0068(9)$ & 0 \\
\hline $\mathrm{Cd} 4$ & $0.011(1)$ & $0.006(1)$ & $0.055(2)$ & 0 & $0.012(1)$ & 0 \\
\hline $\mathrm{Cd} 5$ & $0.0250(8)$ & $0.0266(9)$ & $0.0126(6)$ & $0.0018(7)$ & $0.0041(5)$ & $0.0020(6)$ \\
\hline $\mathrm{Cd} 6$ & $0.0239(8)$ & $0.0203(9)$ & $0.0136(7)$ & $0.0030(7)$ & $0.0028(6)$ & $-0.0001(6)$ \\
\hline $\mathrm{Cd} 7$ & $0.026(1)$ & $0.025(1)$ & $0.014(1)$ & 0 & $0.0001(9)$ & 0 \\
\hline $\mathrm{Cd} 8$ & $0.019(1)$ & $0.028(2)$ & $0.015(1)$ & 0 & $0.0030(9)$ & 0 \\
\hline P1 & $0.009(2)$ & $0.010(2)$ & $0.018(2)$ & $0.0003(7)$ & $0.004(1)$ & $-0.001(1)$ \\
\hline $\mathrm{P} 2$ & $0.009(4)$ & $0.007(3)$ & $0.011(3)$ & 0 & $0.004(2)$ & 0 \\
\hline P4 & $0.013(2)$ & $0.013(2)$ & $0.020(2)$ & $-0.001(1)$ & $0.004(1)$ & $-0.002(1)$ \\
\hline P6 & $0.010(2)$ & $0.011(2)$ & $0.028(4)$ & 0 & $0.005(2)$ & 0 \\
\hline $\mathrm{Na} 1$ & $0.014(2)$ & $0.016(2)$ & $0.016(2)$ & $-0.001(3)$ & $0.006(1)$ & $0.000(2)$ \\
\hline
\end{tabular}



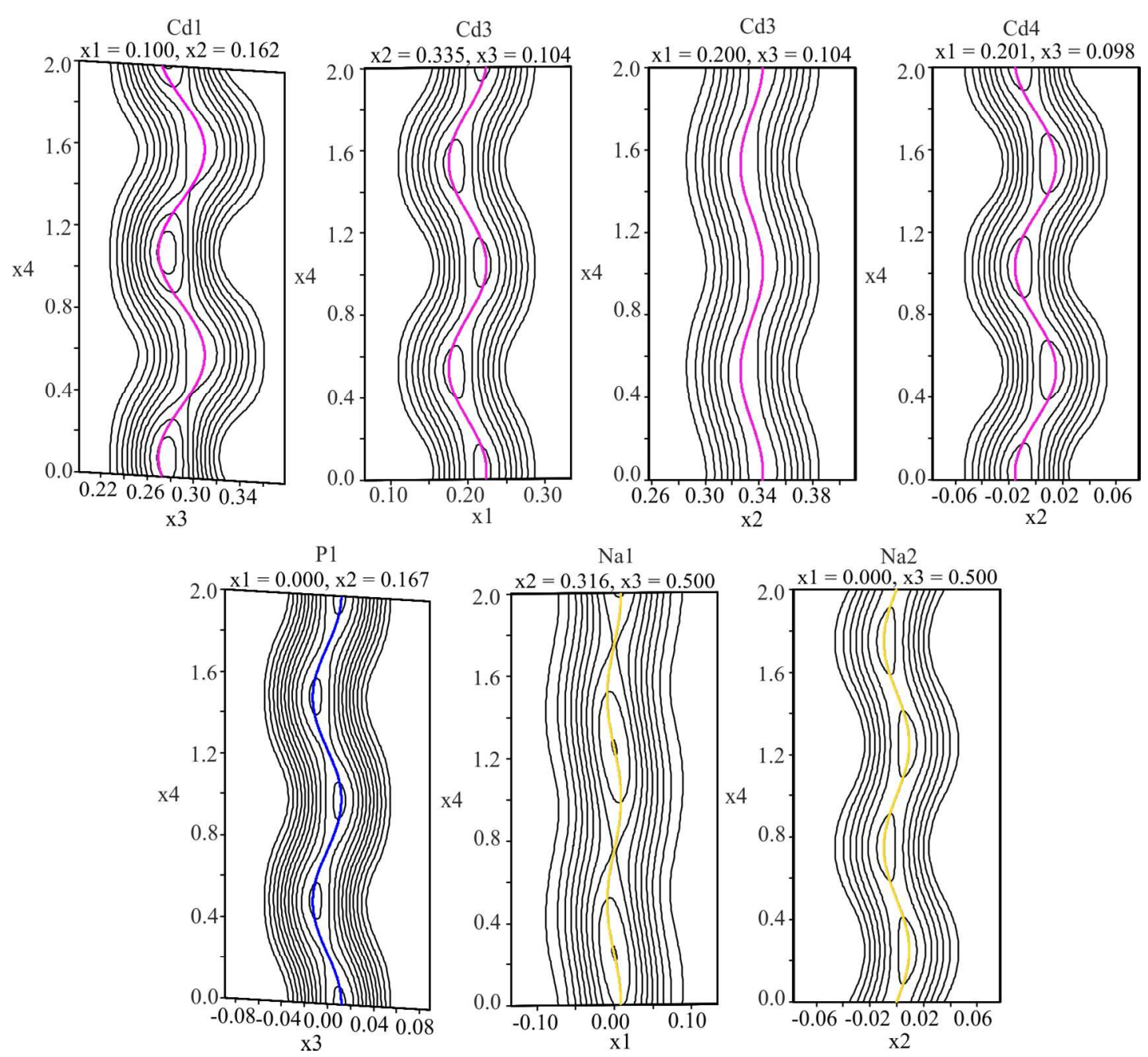

Figure S7. Fourier maps of $\alpha-\mathrm{NaCd}_{4} \mathrm{P}_{3}$ calculated from $130 \mathrm{~K}$ data, space group $C 2 / m(\alpha 0 \gamma) 0 s$. Atom cross sections of selected atoms are given. Contour lines represent differences of $20(\mathrm{Cd})$ and $5 \mathrm{e} \AA^{-3}(\mathrm{P}$ and $\mathrm{Na})$. 
Table S7. Selected bond lengths (in $\AA$ ) of $\alpha-\mathrm{NaCd}_{4} \mathrm{P}_{3}$ and $\beta-\mathrm{NaCd}_{4} \mathrm{P}_{3}$. The bond type is given according the definition in the main text. In the case of the modulated $\alpha$-phase the bond length distribution is given and an average value per bond and for the bond type is calculated. For the definition of Cd-P $\mathrm{P}_{\text {rib }}, \mathrm{Cd}-\mathrm{P}_{\text {handle, }}, \mathrm{Cd}-\mathrm{P}_{\mathrm{sec}}$, and Cd-P $\mathrm{P}_{\text {oct }}$ refer to the main text.

\begin{tabular}{|c|c|c|c|c|c|c|}
\hline \multirow[b]{2}{*}{$\begin{array}{c}\text { Type of } \\
\text { bond }\end{array}$} & \multicolumn{2}{|c|}{$\beta-\mathrm{NaCd}_{4} \mathrm{P}_{3}$} & \multicolumn{4}{|c|}{$\alpha-\mathrm{NaCd}_{4} \mathrm{P}_{3}$} \\
\hline & Bond & $\begin{array}{l}\text { bond } \\
\text { length }\end{array}$ & bond & $\begin{array}{l}\text { Average of } \\
\text { bond length }\end{array}$ & $\begin{array}{l}\text { Bond length } \\
\text { distribution }\end{array}$ & $\begin{array}{l}\text { average of } \\
\text { bond type }\end{array}$ \\
\hline $\mathrm{Cd}-\mathrm{P}_{\text {rib }}$ & Cd1-P1 & $2.5486(8)$ & $\begin{array}{l}\text { Cd1-P3 } \\
\text { Cd1-P5 } \\
\text { Cd2-P4 } \\
\text { Cd2-P6 } \\
\text { Cd3-P3 } \\
\text { Cd3-P5 } \\
\text { Cd4-P4 } \\
\text { Cd4-P6 }\end{array}$ & $\begin{array}{l}2.53(1) \\
2.521(9) \\
2.55(2) \\
2.57(1) \\
2.565(9) \\
2.57(1) \\
2.552(7) \\
2.53(1) \\
\end{array}$ & $\begin{array}{l}2.52-2.54 \\
2.49-2.57 \\
2.49-2.62 \\
2.52-2.63 \\
2.54-2.59 \\
\text { constant } \\
\text { constant } \\
\text { constant } \\
\end{array}$ & 2.55 \\
\hline Cd-P $P_{\text {handle }}$ & $\mathrm{Cd} 2-\mathrm{P} 1$ & $2.571(4)$ & $\begin{array}{l}\text { Cd5-P3 } \\
\text { Cd6-P4 } \\
\text { Cd7-P5 } \\
\text { Cd8-P6 }\end{array}$ & $\begin{array}{l}2.534(10) \\
2.627(9) \\
2.534(12) \\
2.607(14)\end{array}$ & $\begin{array}{l}2.51-2.56 \\
2.61-2.64 \\
\text { constant } \\
2.60-2.61\end{array}$ & 2.575 \\
\hline $\mathrm{Cd}-\mathrm{P}_{\mathrm{sec}}$ & Cd1-P2 & $3.243(2)$ & $\begin{array}{l}\mathrm{Cd} 1-\mathrm{P} 1 \\
\mathrm{Cd} 2-\mathrm{P} 1 \\
\mathrm{Cd} 3-\mathrm{P} 2 \\
\mathrm{Cd} 4-\mathrm{P} 2\end{array}$ & $\begin{array}{l}3.30(4) \\
3.18(5) \\
2.941(8) \\
3.536(8)\end{array}$ & $\begin{array}{l}2.94-3.66 \\
2.81-3.56 \\
\text { constant } \\
\text { constant }\end{array}$ & 3.239 \\
\hline $\mathrm{Cd}-\mathrm{P}_{\text {oct }}$ & Cd2-P2 & $2.7483(6)$ & $\begin{array}{l}\text { Cd5-P1 } \\
\text { Cd5-P2 } \\
\text { Cd6-P1 } \\
\text { Cd6-P2 } \\
\text { Cd7-P1 } \\
\text { Cd7-P2 } \\
\text { Cd8-P1 } \\
\text { Cd8-P2 }\end{array}$ & $\begin{array}{l}2.69(2) \\
2.81(2) \\
2.80(3) \\
2.71(2) \\
2.69(3) \\
2.796(9) \\
2.80(4) \\
2.705(9)\end{array}$ & $\begin{array}{l}2.60-2.79 \\
2.79-2.82 \\
2.70-2.91 \\
2.70-2.72 \\
2.59-2.80 \\
2.792-2.796 \\
2.70-2.91 \\
2.70-2.71\end{array}$ & 2.750 \\
\hline
\end{tabular}




\section{Single crystal X-ray structure data of $\mathrm{NaCd}_{4} \mathrm{As}_{3}$}

Table S8. Summary of structure refinement parameters derived from single crystal X-ray data of all $\mathrm{NaCd}_{4} \mathrm{As}_{3}$ modifications, measured at room temperature (space group $R \overline{3} m$, left column) and at $130 \mathrm{~K}$ (space group $C m(\alpha 0 \gamma) s$ : middle column; space group $C 2 / m(\alpha 0 \gamma) 0 s$ : right column).

\begin{tabular}{|c|c|c|c|}
\hline & $\beta-\mathrm{NaCd}_{4} \mathrm{As}_{3}$ & \multicolumn{2}{|c|}{$\alpha-\mathrm{NaCd}_{4} \mathrm{As}_{3}$} \\
\hline temperature $[\mathrm{K}]$ / space group & $298 / R \overline{3} m$ & $130 / C m(\alpha 0 \gamma) s$ & $130 / C 2 / m(\alpha 0 \gamma) 0 s$ \\
\hline Sum formula & $\mathrm{NaCd}_{4} \mathrm{As}_{3}$ & $\mathrm{NaCd}_{4} \mathrm{As}_{3}$ & $\mathrm{NaCd}_{4} \mathrm{As}_{3}$ \\
\hline Data collection & IPDS-II & IPDS-II & IPDS-II \\
\hline Pearson symbol & $h R 24$ & $m C 48$ & $m C 48$ \\
\hline Molecular weight $[\mathrm{g} / \mathrm{mol}]$ & 697.4 & & 97.4 \\
\hline \multicolumn{4}{|l|}{ Unit cell dimension } \\
\hline$a[\AA]$ & $4.4616(3)$ & \multicolumn{2}{|c|}{$7.7151(8)$} \\
\hline$b[\AA]$ & & \multicolumn{2}{|c|}{ 13.367(1) } \\
\hline$c[\AA]$ & $33.783(3)$ & \multicolumn{2}{|c|}{$11.511(1)$} \\
\hline$\beta[\mathrm{deg}]$ & & \multicolumn{2}{|c|}{$102.905(8)$} \\
\hline Cell volume $\left[\AA^{3}\right]$ & $582.38(7)$ & \multicolumn{2}{|c|}{$1157.1(2)$} \\
\hline$Z$ & 3 & \multicolumn{2}{|c|}{6} \\
\hline$q_{1}$-vector & & \multicolumn{2}{|c|}{$-0.0200 .34$} \\
\hline Calculated density $\left[\mathrm{g} / \mathrm{cm}^{3}\right]$ & 5.9655 & \multicolumn{2}{|c|}{6.0049} \\
\hline Crystal size $\left[\mu \mathrm{m}^{3}\right]$ & $250 \times 400 \times 40$ & \multicolumn{2}{|c|}{ same crystal as for room temperature } \\
\hline Transmníssion ratio $[\mathrm{max} / \mathrm{min}]$ & 15.57 & 13.38 & 14.54 \\
\hline Detector distance $[\mathrm{mm}]$ & 80 & \multicolumn{2}{|r|}{80} \\
\hline Exposure time [min] & 0.3 & \multicolumn{2}{|c|}{0.1} \\
\hline$\theta$ range $[\mathrm{deg}]$, increment $[\mathrm{deg}]$ & $0-180,1.0$ & \multicolumn{2}{|c|}{$0-180,1.0$} \\
\hline Integration parameters (A, B, EMS) & $12.2,2.7,0.016$ & \multicolumn{2}{|c|}{$9.5,1.6,0.010$} \\
\hline Absorption coefficient $\left[\mathrm{mm}^{-1}\right]$ & 23.71 & \multicolumn{2}{|c|}{23.87} \\
\hline $\mathrm{F}(000)$ & 906 & \multicolumn{2}{|c|}{1812} \\
\hline$\theta$ range for data collection $[\mathrm{deg}]$ & $3.63-31.91$ & \multicolumn{2}{|c|}{$3.6-32.12$} \\
\hline Range in $h k l$ & $\pm 6, \pm 6,0-49$ & \multicolumn{2}{|c|}{ $\pm 11, \pm 19, \pm 17$} \\
\hline Total no. of reflections & 1353 & 35411 & 35411 \\
\hline Independent reflections / $\mathrm{R}_{\text {int }}$ & $172 / 0.0657$ & $11345 / 0.103$ & $5794 / 0.102$ \\
\hline Reflections with $\mathrm{I}>3 \sigma(\mathrm{I}) / \mathrm{R}_{\sigma}$ & $169 / 0.0444$ & 4609 / 0.0741 & $2685 / 0.0678$ \\
\hline No. of data / param & $172 / 15$ & 11345 / 194 & $5794 / 134$ \\
\hline GOF on $\mathrm{F}^{2}$ & 3.68 & 1.35 & 1.97 \\
\hline $\mathrm{R} 1 / w \mathrm{R} 2[\mathrm{I}>3 \sigma(\mathrm{I})]$ & $0.0454 / 0.1097$ & $0.0481 / 0.1082$ & $0.0728 / 0.1704$ \\
\hline $\mathrm{R} 1 / w \mathrm{R} 2$ (all data) & $0.0485 / 0.1098$ & $0.1065 / 0.1270$ & $0.1254 / 0.1918$ \\
\hline $\mathrm{R} 1 / w \mathrm{R} 2[\mathrm{I}>3 \sigma(\mathrm{I})]$ (main) & - & $0.0415 / 0.0956$ & $0.0711 / 0.1676$ \\
\hline $\mathrm{R} 1 / w \mathrm{R} 2$ (all data) (main) & - & $0.0638 / 0.1003$ & $0.1004 / 0.1826$ \\
\hline $\mathrm{R} 1 / w \mathrm{R} 2[\mathrm{I}>3 \sigma(\mathrm{I})]$ (satellites) & - & $0.0705 / 0.1624$ & $0.0780 / 0.1821$ \\
\hline R1 / wR2 (all data) (satellites) & - & $0.2115 / 0.2235$ & $0.1865 / 0.2262$ \\
\hline Extinction coefficient & $1530(170)$ & $1520(40)$ & $2250(90)$ \\
\hline Largest diff peak/hole $\left[\mathrm{e} / \AA^{3}\right]$ & $1.84 /-2.39$ & $3.32 /-2.41$ & $6.05 /-4.95$ \\
\hline Twinning fraction & - & $0.55(4)$ & - \\
\hline
\end{tabular}




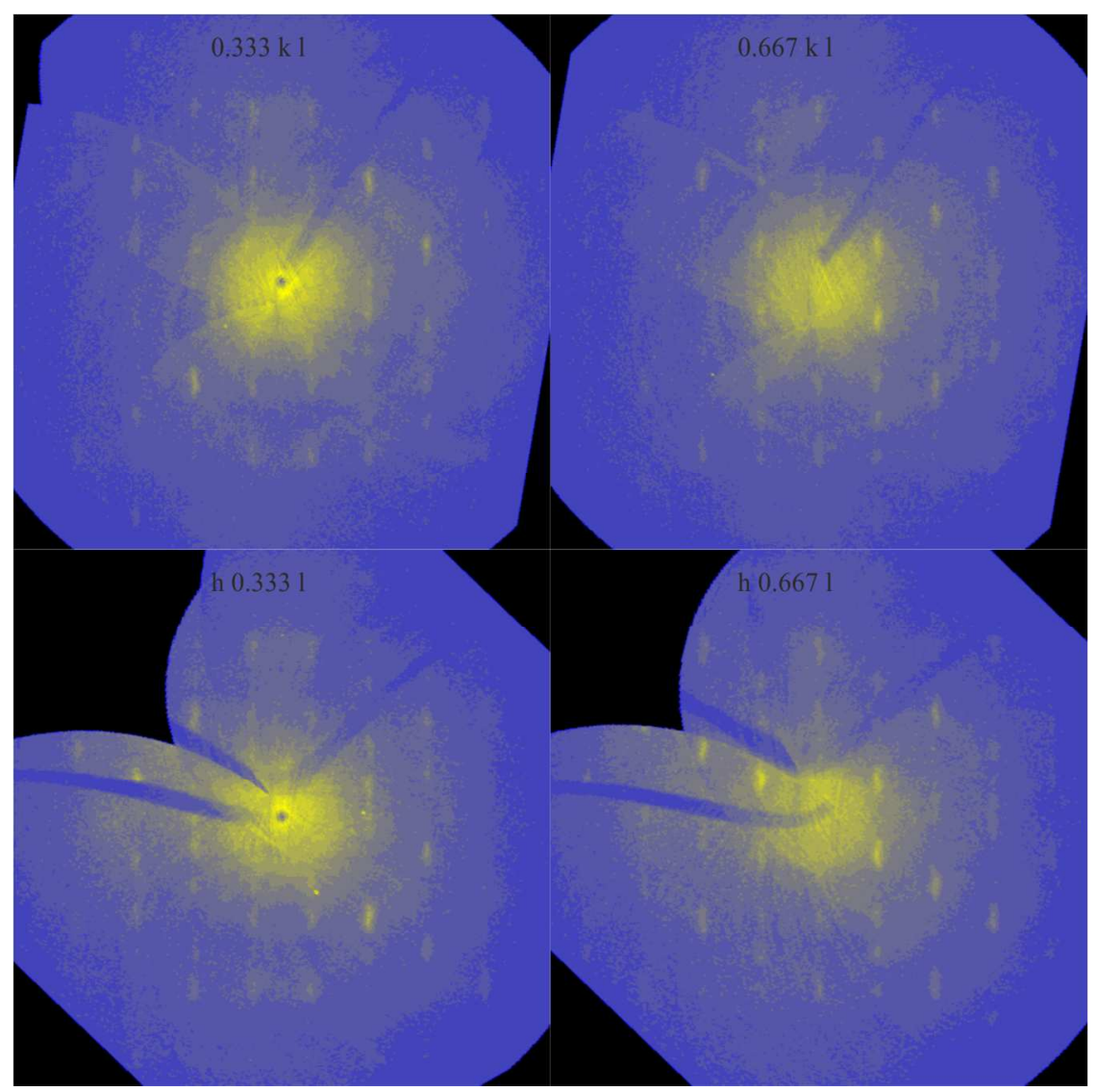

Figure S8. Reciprocal space representation of $\beta-\mathrm{NaCd}_{4} \mathrm{As}_{3}$, derived from room temperature $\mathrm{X}$ ray data. A rhombohedral cell with cell parameters $a=4.4616(3) \AA, c=33.783(3) \AA$ (space group: $R \overline{3} \mathrm{~m}$ ) was used to build up these pictures. Wave-like diffuse scattering intensities are present at $(0.333 k l),(0.667 k l),(h 0.333 l)$, and $(h 0.667 l)$ layers. All diffuse intensity is neglected in the room temperature refinements. 
Table S9. Crystal structure parameters and atomic displacements $\left(\AA^{2}\right)$, derived from X-ray single crystal X-ray data of $\beta-\mathrm{NaCd}_{4} \mathrm{As}_{3}$, measured at room temperature (space group $R \overline{3} \mathrm{~m}$ ). The anisotropic displacement factor exponent takes the form: $-2 \pi^{2}\left[\left(\mathrm{ha}^{*}\right) 2 U_{11}+\ldots+2 \mathrm{hka} \cdot \mathrm{b} \cdot U_{12}\right] . U_{e q}$ is defined as one third of the trace of the orthogonalized $U_{i j}$ tensor.

\begin{tabular}{|c|c|c|c|c|c|c|}
\hline$\beta-\mathrm{NaCd}_{4} \mathrm{As}_{3}$ & \multicolumn{2}{|c|}{ Wyckoff position } & $x$ & $y$ & $z$ & $U_{i s o} / U_{e q}$ \\
\hline $\mathrm{Cd} 1$ & \multicolumn{2}{|c|}{$6 c$} & 0 & 0 & $0.09583(5)$ & $0.0318(7)$ \\
\hline As1 & \multicolumn{2}{|c|}{$6 c$} & 0 & 0 & $0.22070(5)$ & $0.0121(7)$ \\
\hline $\mathrm{Cd} 2$ & \multicolumn{2}{|c|}{$6 c$} & 0 & 0 & $0.29896(4)$ & $0.0300(7)$ \\
\hline $\mathrm{Na} 1$ & \multicolumn{2}{|c|}{$3 b$} & 0 & 0 & $1 / 2$ & $0.026(3)$ \\
\hline \multirow[t]{2}{*}{ As2 } & \multicolumn{2}{|c|}{$3 a$} & 0 & 0 & 0 & $0.0157(8)$ \\
\hline & $U_{11}$ & $U_{22}$ & $U_{33}$ & $U_{12}$ & $U_{13}$ & $U_{23}$ \\
\hline $\mathrm{Cd} 1$ & $0.0127(8)$ & $0.0127(8)$ & $0.070(1)$ & $0.0064(4)$ & 0 & 0 \\
\hline As 1 & $0.0098(8)$ & $0.0098(8)$ & $0.017(1)$ & $0.0049(4)$ & 0 & 0 \\
\hline $\mathrm{Cd} 2$ & $0.0361(9)$ & $0.0361(9)$ & $0.018(1)$ & $0.0180(5)$ & 0 & 0 \\
\hline $\mathrm{Na} 1$ & $0.035(4)$ & $0.035(4)$ & $0.008(5)$ & $0.018(2)$ & 0 & 0 \\
\hline As2 & $0.014(1)$ & $0.014(1)$ & $0.018(2)$ & $0.0072(5)$ & 0 & 0 \\
\hline
\end{tabular}

Table S10. Selected interatomic distances $(\AA)$ derived from single crystal X-ray data of $\beta$ $\mathrm{NaCd}_{4} \mathrm{As}_{3}$, measured at room temperature (space group: $R \overline{3} m$ ). All distances of the first coordination sphere including standard deviations are given.

\begin{tabular}{|c|c|c|c|c|c|c|c|}
\hline atom 1 & & atom 2 & distance $[\AA]$ & atom 1 & & atom 2 & distance $[\AA]$ \\
\hline \multirow[t]{3}{*}{$\overline{C d} 1$} & 3 & As1 & $2.6377(5)$ & $\mathrm{Cd} 2$ & & As1 & $2.644(2)$ \\
\hline & & As2 & $3.237(2)$ & & 3 & As2 & $2.8256(6)$ \\
\hline & 3 & $\mathrm{Na} 1$ & $3.516(1)$ & $\mathrm{Na} 1$ & 6 & As1 & $3.157(1)$ \\
\hline \multirow[t]{3}{*}{ As1 } & 3 & $\mathrm{Cd} 1$ & $2.6377(5)$ & & 6 & $\mathrm{Cd} 1$ & $3.516(1)$ \\
\hline & & $\mathrm{Cd} 2$ & $2.644(2)$ & As2 & 6 & $\mathrm{Cd} 2$ & $2.8256(6)$ \\
\hline & 3 & $\mathrm{Na} 1$ & $3.157(1)$ & & & $\mathrm{Cd} 1$ & $3.237(2)$ \\
\hline
\end{tabular}




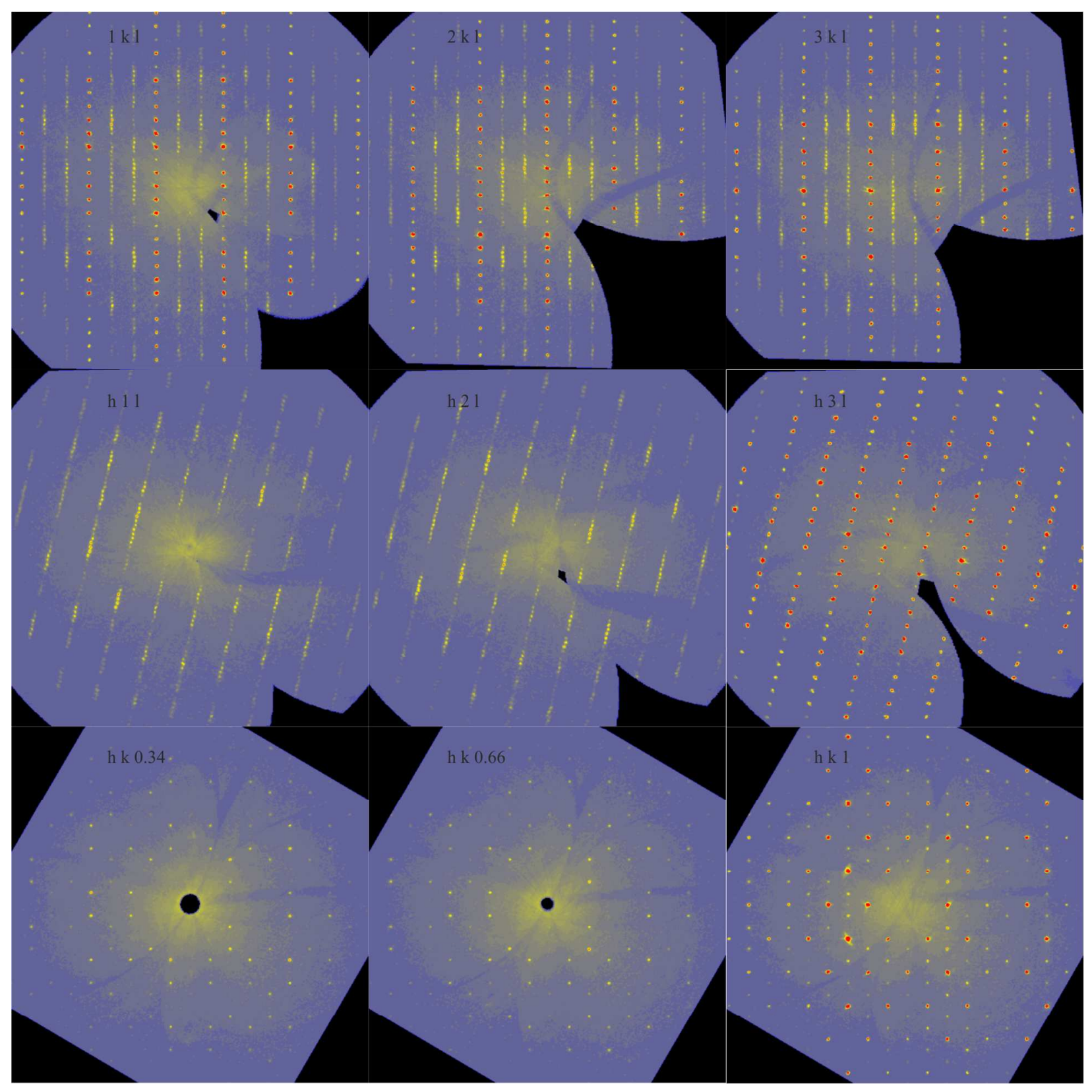

Figure S9. Reciprocal space representation of $\alpha-\mathrm{NaCd}_{4} \mathrm{As}_{3}$, derived from low temperature X-ray data $(130 \mathrm{~K})$. A monoclinic cell with cell parameters $a=7.7151(8) \AA, b=13.367(1) \AA, c=$ 11.511(1) $\AA, \beta=102.905(8)^{\circ}$ was used to create these pictures (space group $C m(\alpha 0 \gamma) s$ ). Areas of diffuse scattering which were present at room temperature (see Figure S8) show discrete Bragg reflections now. 
Table S11. Crystal structure parameters and atomic displacements $\left(\AA^{2}\right)$, derived from X-ray single crystal X-ray data of $\alpha-\mathrm{NaCd}_{4} \mathrm{As}_{3}$, measured at low temperature $(130 \mathrm{~K})$. Wyckoff position, atomic coordinates, isotropic and anisotropic displacement parameters $\left(\AA^{2}\right)$ for $\alpha$ $\mathrm{NaCd}_{4} \mathrm{As}_{3}$ are given (space group $\left.\mathrm{Cm}(\alpha 0 \gamma) s\right)$. The anisotropic displacement factor exponent takes the form: $-2 \pi^{2}\left[\left(\mathrm{ha}^{*}\right) 2 U_{11}+\ldots+2 \mathrm{hka} \cdot \mathrm{b} \cdot U_{12}\right] . U_{e q}$ is defined as one third of the trace of the orthogonalized $U_{i j}$ tensor.

\begin{tabular}{|c|c|c|c|c|c|c|}
\hline$\alpha-\mathrm{NaCd}_{4} \mathrm{As}_{3}$ & \multicolumn{2}{|c|}{ Wyckoff position } & $x$ & $y$ & $z$ & $U_{i s o} * / U_{e q}$ \\
\hline $\mathrm{Cd} 1$ & \multicolumn{2}{|c|}{$4 b$} & $0.1830(2)$ & $0.1664(1)$ & $0.0487(2)$ & $0.0191(4)$ \\
\hline $\mathrm{Cd} 2$ & \multicolumn{2}{|c|}{$4 b$} & $0.3745(2)$ & $0.1666(1)$ & $0.6244(2)$ & $0.0185(4)$ \\
\hline $\mathrm{Cd} 3$ & \multicolumn{2}{|c|}{$2 a$} & $0.6870(2)$ & 0 & $0.0616(2)$ & $0.0122(4)$ \\
\hline $\mathrm{Cd} 4$ & \multicolumn{2}{|c|}{$2 a$} & $0.8806(3)$ & 0 & $0.6383(2)$ & $0.0191(6)$ \\
\hline $\mathrm{Cd} 5$ & \multicolumn{2}{|c|}{$4 b$} & $0.0747(2)$ & $0.3282(2)$ & $0.2374(1)$ & $0.0143(3)$ \\
\hline $\mathrm{Cd} 6$ & \multicolumn{2}{|c|}{$4 b$} & $0.4767(2)$ & $0.3377(2)$ & $0.4432(2)$ & $0.0193(4)$ \\
\hline $\mathrm{Cd} 7$ & \multicolumn{2}{|c|}{$2 a$} & $0.0894(2)$ & 0 & $0.2368(2)$ & $0.0139(4)$ \\
\hline $\mathrm{Cd} 8$ & \multicolumn{2}{|c|}{$2 a$} & $0.4913(3)$ & 0 & $0.4424(2)$ & $0.0194(5)$ \\
\hline As1 & \multicolumn{2}{|c|}{$4 b$} & $0.2810(3)$ & $0.1667(2)$ & $0.3420(2)$ & $0.0099(3)$ \\
\hline As2 & \multicolumn{2}{|c|}{$2 a$} & $0.7793(3)$ & 0 & $0.3340(2)$ & $0.0093(7)$ \\
\hline As3 & \multicolumn{2}{|c|}{$4 b$} & $0.0015(3)$ & $0.3338(1)$ & $0.0005(2)$ & $0.0096(3) *$ \\
\hline As4 & \multicolumn{2}{|c|}{$4 b$} & $0.0603(2)$ & $0.1674(1)$ & $0.6782(2)$ & $0.0048(2)$ \\
\hline As5 & \multicolumn{2}{|c|}{$2 a$} & $-0.0013(3)$ & 0 & $-0.0010(3)$ & $0.0098(4)^{*}$ \\
\hline As6 & \multicolumn{2}{|c|}{$2 a$} & $0.5583(3)$ & 0 & $0.6770(2)$ & $0.0042(3)$ \\
\hline $\mathrm{Na} 1$ & \multicolumn{2}{|c|}{$4 b$} & $0.281(1)$ & $0.332(1)$ & $0.8429(6)$ & $0.0095(6)^{*}$ \\
\hline \multirow[t]{2}{*}{$\mathrm{Na} 2$} & \multicolumn{2}{|c|}{$2 a$} & $0.290(1)$ & 0 & $0.8435(8)$ & $0.02 *$ \\
\hline & $U_{11}$ & $U_{22}$ & $U_{33}$ & $U_{12}$ & $U_{13}$ & $U_{23}$ \\
\hline $\mathrm{Cd} 1$ & $0.0099(5)$ & $0.0096(6)$ & $0.0393(9)$ & $0.0000(4)$ & $0.0088(5)$ & $0.0005(5)$ \\
\hline $\mathrm{Cd} 2$ & $0.0096(5)$ & $0.0079(6)$ & $0.0390(9)$ & $0.0003(4)$ & $0.0080(5)$ & $0.0003(6)$ \\
\hline $\mathrm{Cd} 3$ & $0.0068(6)$ & $0.0036(6)$ & $0.0277(8)$ & 0 & $0.0069(5)$ & 0 \\
\hline $\mathrm{Cd} 4$ & $0.0077(6)$ & $0.0016(7)$ & $0.051(1)$ & 0 & $0.0120(7)$ & 0 \\
\hline $\mathrm{Cd} 5$ & $0.0207(5)$ & $0.0192(6)$ & $0.0029(3)$ & $-0.0009(4)$ & $0.0023(3)$ & $0.0011(4)$ \\
\hline $\mathrm{Cd} 6$ & $0.0215(6)$ & $0.0191(7)$ & $0.0172(5)$ & $0.0017(4)$ & $0.0039(4)$ & $0.0002(4)$ \\
\hline $\mathrm{Cd} 7$ & $0.0169(7)$ & $0.0204(9)$ & $0.0034(5)$ & 0 & $0.0003(5)$ & 0 \\
\hline $\mathrm{Cd} 8$ & $0.0165(6)$ & $0.024(1)$ & $0.0175(7)$ & 0 & $0.0038(5)$ & 0 \\
\hline As1 & $0.0077(4)$ & $0.0076(6)$ & $0.0147(6)$ & $-0.0004(2)$ & $0.0032(4)$ & $-0.0004(3)$ \\
\hline As2 & $0.0081(9)$ & $0.005(1)$ & $0.015(1)$ & 0 & $0.0042(9)$ & 0 \\
\hline As4 & $0.0049(3)$ & $0.0019(3)$ & $0.0083(4)$ & $-0.0003(2)$ & $0.0033(2)$ & $0.0004(3)$ \\
\hline As6 & $0.0043(5)$ & $0.0019(5)$ & $0.0072(5)$ & 0 & $0.0030(3)$ & 0 \\
\hline
\end{tabular}



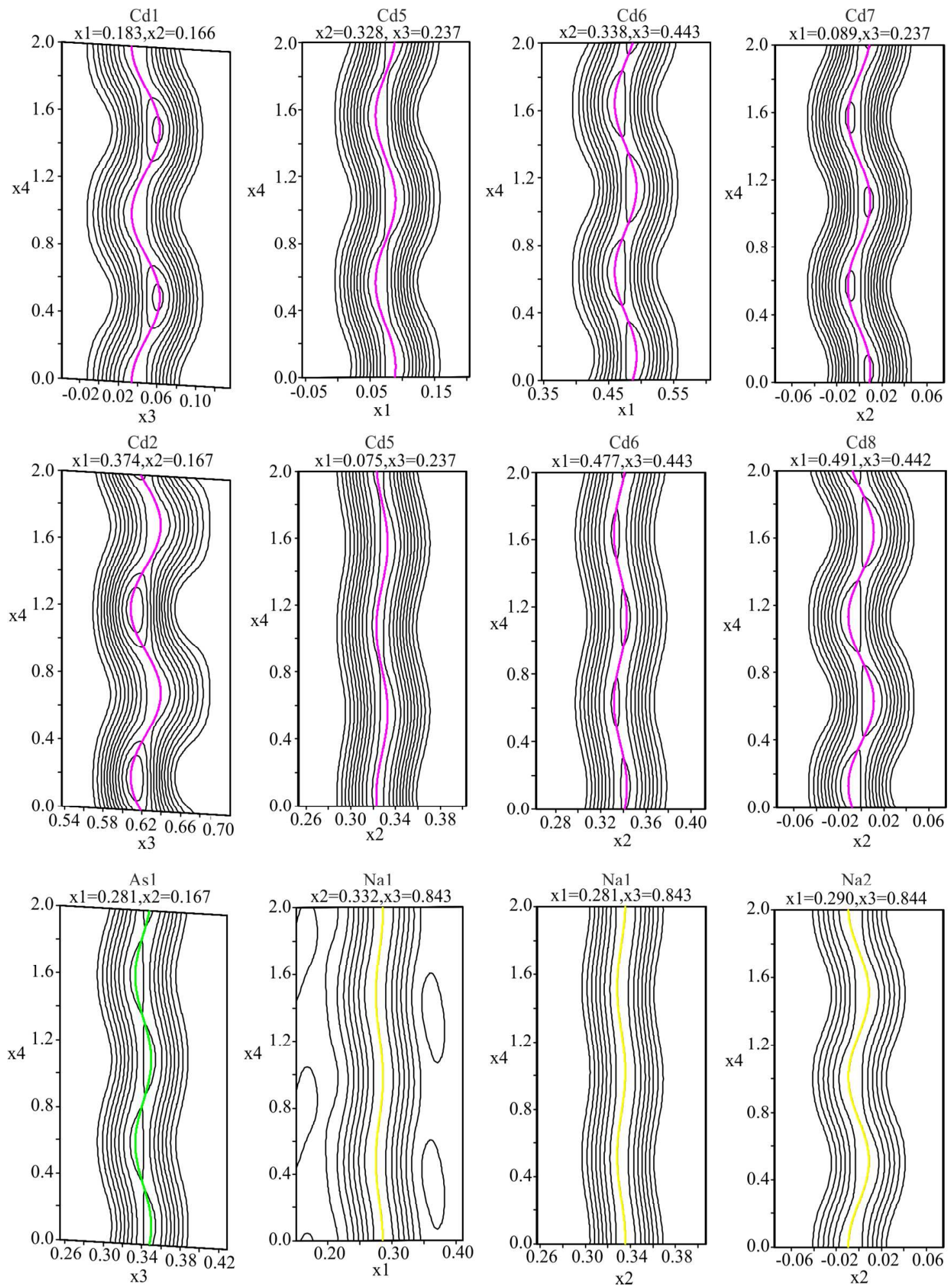

Figure S10. Fourier maps of $\alpha-\mathrm{NaCd}_{4} \mathrm{As}_{3}$, space group $C m(\alpha 0 \gamma) s$, derived from single crystal Xray data measured at $130 \mathrm{~K}$. Atom cross sections of selected atoms are given. Contour lines represent differences of $20(\mathrm{Cd}$ and $\mathrm{As})$ and $5 \mathrm{e}^{-3}(\mathrm{Na})$. 

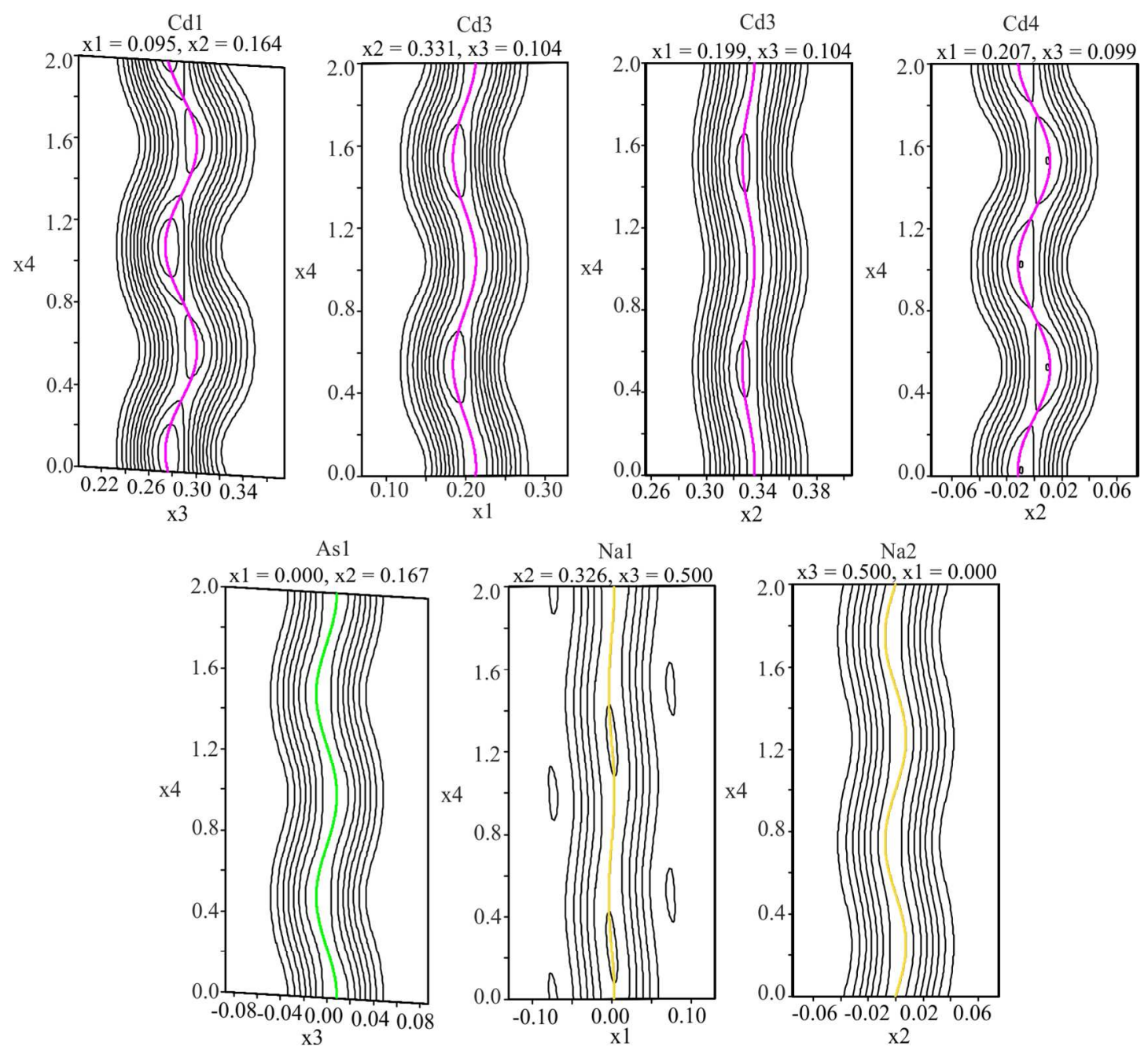

Figure S11. Fourier maps of $\alpha-\mathrm{NaCd}_{4} \mathrm{As}_{3}$, space group $C 2 / m(\alpha 0 \gamma) 0 s$, derived from single crystal $\mathrm{X}$-ray data measured at $130 \mathrm{~K}$. Atom cross sections of selected atoms are given. Contour lines represent differences of $20(\mathrm{Cd}$ and $\mathrm{As})$ and $5 \mathrm{e}^{-3}(\mathrm{Na})$. 

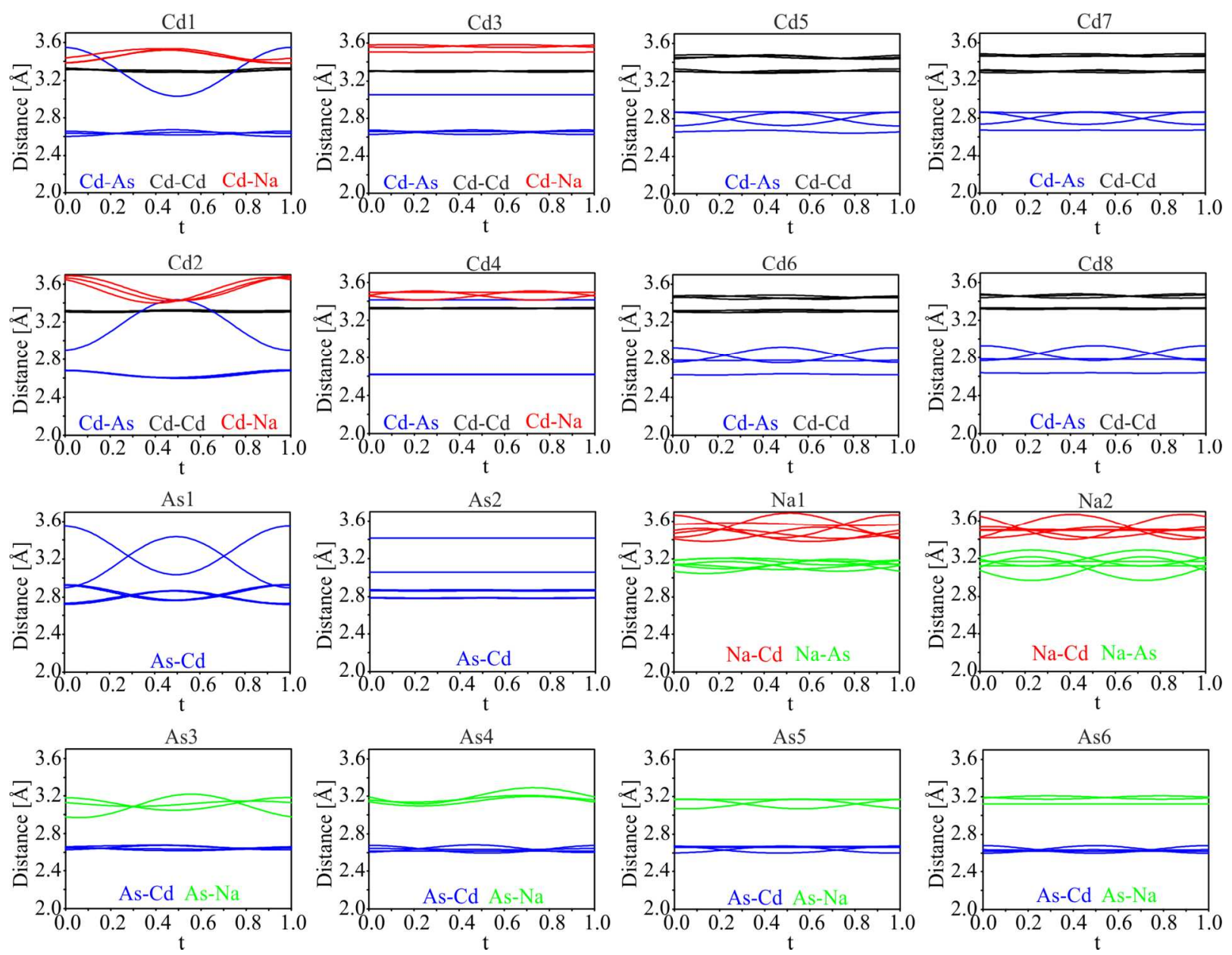

Figure S12. All distance plots of $\alpha-\mathrm{NaCd}_{4} \mathrm{As}_{3}$ derived from single crystal data (130 K; space group

$\mathrm{Cm}(\alpha 0 \gamma) s)$. 
Raman spectrum of $\mathrm{NaCd}_{4} \mathrm{As}_{3}$ :

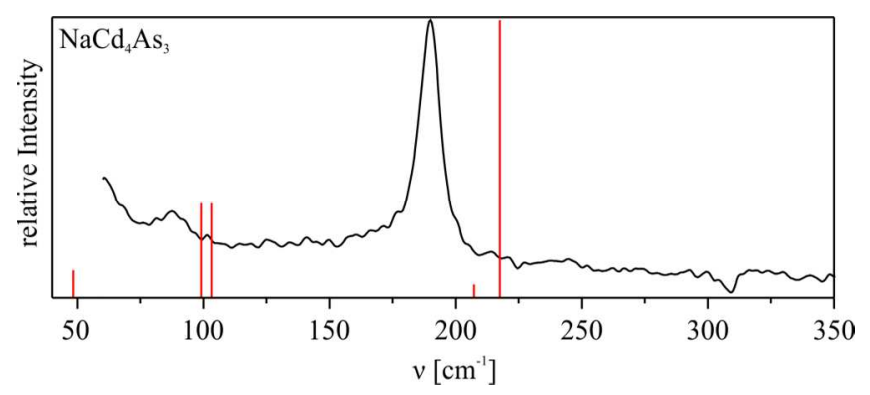

Figure S13. The experimental Raman spectrum (black) and calculated Raman bands (red) of $\beta$ $\mathrm{NaCd}_{4} \mathrm{As}_{3}$. Only the range where signals appear is depicted.

Additional figures concerning the electronic structure of both phases:

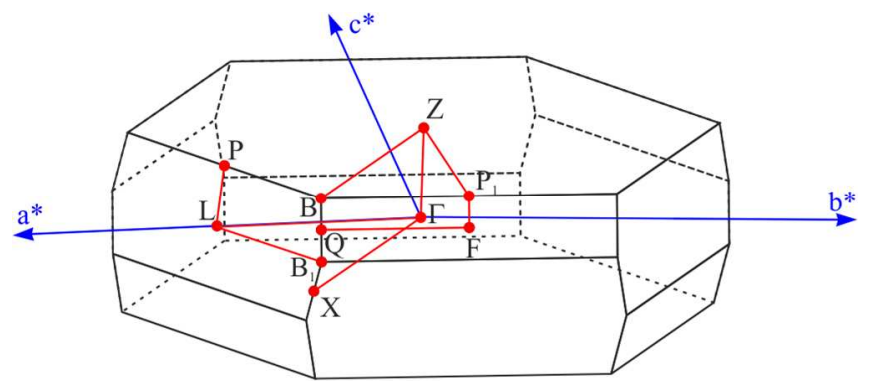

Figure S14. Brillouin zone with path used for the quantum chemical calculations of $\beta-A T_{4} P n_{3}$ phases. 


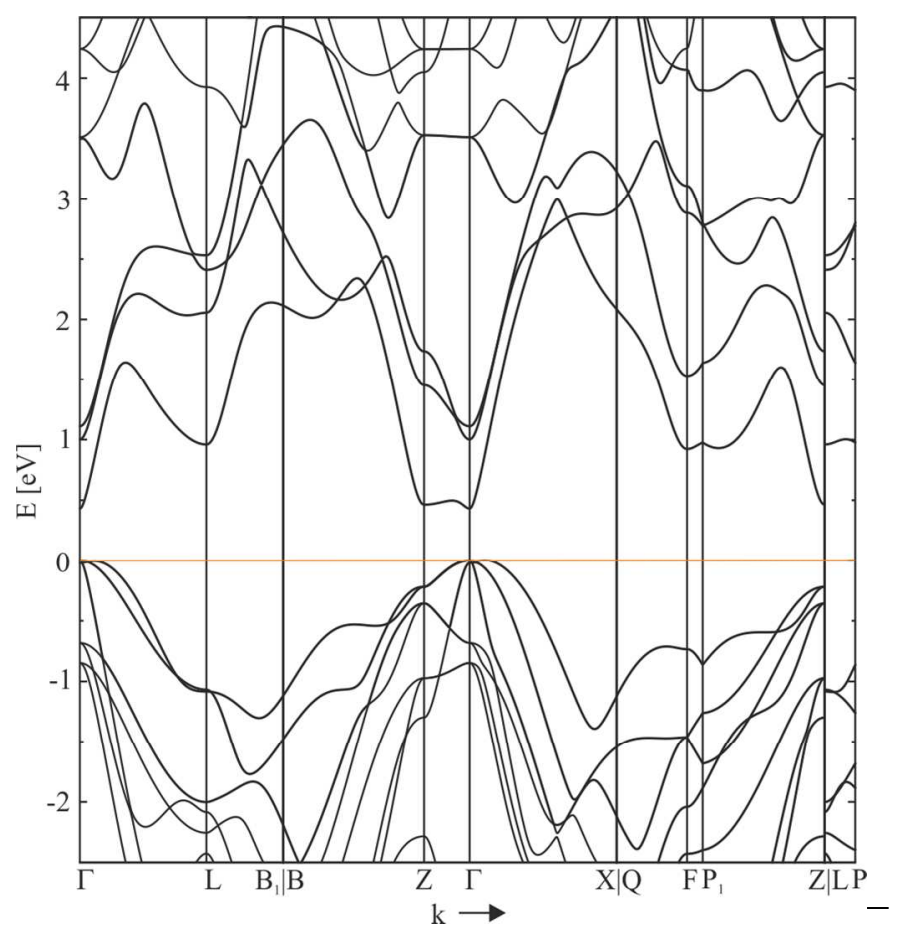

Figure S15. Band structure of $\beta-\mathrm{NaCd}_{4} \mathrm{As}_{3}$ calculated within the framework of DFT.

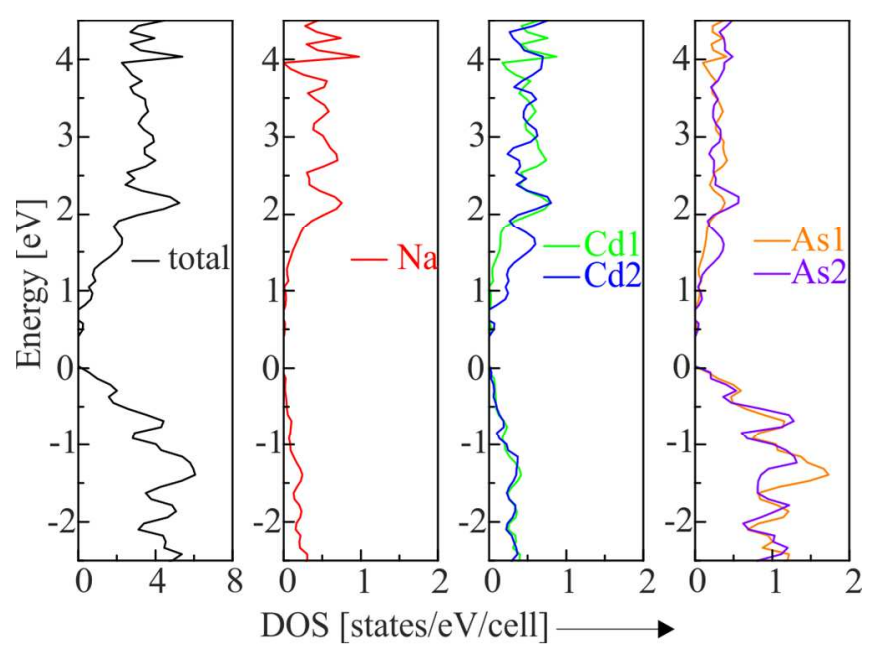

Figure S16. DOS of $\beta-\mathrm{NaCd}_{4} \mathrm{As}_{3}$ calculated within the framework of DFT. 